\title{
Aprotinin is safe in pediatric patients undergoing cardiac surgery
}

\author{
Carl L. Backer, MD, ${ }^{a}$ Angela M. Kelle, BS, ${ }^{\text {a }}$ Robert D. Stewart, MD, MPH, ${ }^{\text {a }}$ Sunitha C. Suresh, ${ }^{a}$ Farah N. Ali, MD, ${ }^{\text {b }}$
} Richard A. Cohn, MD, ${ }^{\mathrm{b}}$ Roopa Seshadri, PhD, ${ }^{\mathrm{c}}$ and Constantine Mavroudis, MDa

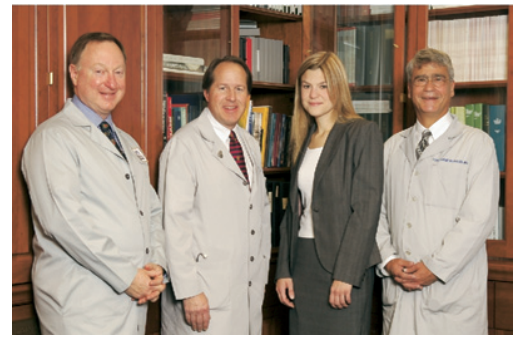

Drs Cohn, Backer, Ms Kelle, and Dr Mavroudis (left to right)
From the Division of Cardiovascular-Thoracic Surgery, Department of Surgery ${ }^{\mathrm{a}}$ and the Division of Kidney Diseases, Department of Pediatrics, ${ }^{\mathrm{b}}$ Northwestern University Feinberg School of Medicine, Chicago, Ill; Children's Memorial Hospital, and the Department of Pediatrics and Preventive Medicine, Northwestern University Feinberg School of Medicine, and the Mary Ann and J. Milburn Smith Child Health Research Program, Children's Memorial Research Center, ${ }^{\mathrm{c}}$ Chicago, Ill.

Read at the Eighty-seventh Annual Meeting of The American Association for Thoracic Surgery, Washington, DC, May 5-9, 2007.

Received for publication May 16, 2007; revisions received July 19, 2007; accepted for publication Aug 7, 2007.

Address for reprints: Carl L. Backer, MD, Division of Cardiovascular-Thoracic Surgery, Children's Memorial Hospital, 2300 Children's Plaza, mc 22, Chicago, IL 60614 (E-mail: cbacker@childrensmemorial.org).

J Thorac Cardiovasc Surg 2007;134:1421-8

$0022-5223 / \$ 32.00$

Copyright (C) 2007 by The American Association for Thoracic Surgery

doi:10.1016/j.jtcvs.2007.08.006
Objective: Aprotinin, a serine protease inhibitor, decreases transfusion requirements and inflammatory response after cardiopulmonary bypass. This study was done to determine whether aprotinin is associated with adverse outcomes, particularly mortality and acute kidney failure, in pediatric patients $(<18$ years of age) undergoing cardiopulmonary bypass.

Methods: We compared a cohort of all pediatric cardiopulmonary bypass operations from 1994-1999, when aprotinin was not used $(\mathrm{n}=1230)$, with a cohort from 2000-2006, when all patients received high-dose aprotinin $(n=1251)$. Primary end points were operative and late mortality, acute kidney failure, need for dialysis, and neurologic complications. Association of aprotinin with primary end points was assessed by means of univariate analysis, multivariate logistic regression, and Cox regression analysis, where appropriate.

Results: The aprotinin group was younger (mean age, $3.49 \pm 1.84$ vs $3.64 \pm 4.75$ years; $P=.019)$ and had a higher Aristotle score $(7.8 \pm 2.3$ vs $7.2 \pm 2.6, P<.001)$. Univariate and multivariate analysis showed no significant difference between the no-aprotinin and aprotinin groups for operative mortality (55 [4.5\%] vs 47 [3.8\%], $P=.508)$, acute kidney failure $(68[6.0 \%]$ vs $69[5.7 \%], P=.77)$, need for temporary dialysis $(6[0.49 \%]$ vs 12 [0.96\%], $P=.17)$, or neurologic complications $(14[1.1 \%]$ vs 17 [1.4\%], $P=.62)$. By means of Cox regression analysis, aprotinin had no influence on late mortality ( 24 vs 10 deaths, $P=.078$ ).

Conclusion: In this retrospective cohort study of pediatric patients undergoing cardiopulmonary bypass, there was no association between the use of aprotinin and acute kidney failure, need for dialysis, neurologic complications, and operative or late mortality. We continue to use aprotinin for all pediatric patients undergoing cardiopulmonary bypass.

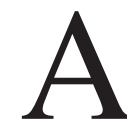
protinin (Trasylol; Bayer Pharmaceutical, West Haven, Conn) is an antifibrinolytic serine protease inhibitor purified from bovine lung. Aprotinin was approved by the US Food and Drug Administration to reduce perioperative blood loss in high-risk patients undergoing cardiopulmonary bypass (CPB) for coronary artery bypass grafting in $1993 .{ }^{1}$ Aprotinin reduces bleeding by delaying the rapid plasmin-mediated lysis of the fibrin clot. Several randomized, prospective, placebo-controlled, carefully performed trials on aprotinin use have shown that it reduces requirements for blood transfusion in adult cardiac surgery. ${ }^{2-4}$ Aprotinin has also been shown to decrease the inflammatory response to CPB. ${ }^{5,6}$ After completing an internal study that demonstrated that aprotinin reduced operative closure time and blood product use after pediatric cardiac bypass, our center began routinely using aprotinin in all of our patients undergoing CPB in early $2000 .^{7}$ Several other pediatric cardiac surgery centers have shown that the use of aprotinin in pediatric 

Abbreviations and Acronyms
$\mathrm{CPB}=$ cardiopulmonary bypass
DHCA $=$ deep hypothermic circulatory arrest

patients undergoing CPB is associated with decreased use of blood products and cost savings from decreased operative time. ${ }^{8,9}$

Recently, the safety of aprotinin use in adult cardiac surgery has been called into question, particularly in 2 separate reports published by Mangano and colleagues. ${ }^{10,11}$ They reported that aprotinin use was associated with increased risk of perioperative acute kidney failure, cerebral vascular accidents, and long-term mortality. Both studies have elicited numerous letters to the editor and editorials. ${ }^{12-14}$

The purpose of our study was to determine whether the use of aprotinin was associated with adverse outcomes, particularly mortality and impairment of kidney function, in pediatric patients $(<18$ years of age) undergoing CPB. We also analyzed the patients with regard to postoperative newonset neurologic injury. The conduct of this study was facilitated by our change in treatment protocols to include aprotinin in all of our patients undergoing CPB in early 2000. This study was not directed at the efficacy of aprotinin; it was limited to the safety issues recently raised.

\section{Materials and Methods}

This was a retrospective, nonrandomized cohort study. The Institutional Review Board at Children's Memorial Hospital, Chicago, Illinois, approved this study and granted a waiver of informed consent on May 4, 2006. We identified patients through the computerized database for cardiac surgical patients at Children's Memorial Hospital, which was established in 1990. We compared all pediatric patients having CPB for the 6-year period before use of aprotinin (1994-1999) with a cohort of patients who all received aprotinin (2000-2006). The aprotinin protocol that we used is shown in Table 1 . The primary outcome measures evaluated were operative and late mortality, biochemical acute kidney failure, and the need for temporary dialysis. Operative mortality was defined as death within 30 days of an operation or within the primary hospi-

\begin{tabular}{l} 
TABLE 1. Aprotinin protocol at Children's Memorial \\
Hospital \\
- $1-\mathrm{mL}$ test dose after arterial line placed \\
- $171.5 \mathrm{~mL} / \mathrm{m}^{2}$ patient loading and pump prime dose (maximum, \\
$200 \mathrm{~mL}$ ) \\
- $40 \mathrm{~mL} / \mathrm{m}^{2} / \mathrm{h}$ infusion through bypass run and for $1 \mathrm{~h}$ \\
postoperatively (maximum, $50 \mathrm{~mL} / \mathrm{h}$ ) \\
- $1 \mathrm{~mL}=10,000 \mathrm{KIU}$ \\
\hline
\end{tabular}

talization (Society of Thoracic Surgeons and European Association for Cardiothoracic Surgery definition). ${ }^{15}$ Biochemical acute kidney failure was defined as an increase in serum creatinine levels to twice or more than the preoperative level (personal e-mail communication with J. P. Jacobs, Multi-Societal Pediatric and Congenital Cardiac Database Taskforce, 2007). The serum creatinine value selected for analysis was the peak value in the first postoperative 72 hours. ${ }^{16}$ The need for temporary dialysis was defined as any patient having placement of an intravenous dialysis catheter or peritoneal dialysis catheter that was used for dialysis at any time during the hospital stay.

Demographic and clinical characteristics were summarized by using means and standard deviations for continuous variables and frequencies for categorical variables and compared between the 2 study groups by using $t$ tests and $\chi^{2}$ tests. All outcomes of interest were as follows: biochemical acute kidney failure, postoperative temporary dialysis, operative mortality, and late mortality are binary. In addition to aprotinin, predictors studied included age, sex, body surface area, operative status (emergency status vs nonemergency status), Aristotle score, deep hypothermic circulatory arrest (DHCA; yes vs no), preoperative ventilator support (yes vs no), prior open cardiothoracic operations (yes vs no), CPB time, aortic crossclamp time, and preoperative serum creatinine level. The data consisted of multiple operations per patient over the study period. The repeated nature of the data was taken into consideration in the regression analyses.

A Cox regression frailty model (Therneau) with a random effect for patient was used to determine predictors of late mortality. ${ }^{17}$ Time at risk was defined by using the Anderson-Gill approach. Time at risk for patients with multiple operations was from one surgical date to the next. Follow-up times were censored at April 27, 2007. A generalized linear model for binary outcome (Liang and Zeger) with logit link was used to determine predictors of the other 3 outcomes. Repeated measures were modeled by using compound symmetry structure. ${ }^{18}$ The overall strategy was to use results from univariate associations as a data reduction tool to identify candidates for a multiple-predictor model. Univariate models included each predictor one at a time, controlling for aprotinin. A $P$ value of .1 determined inclusion in a multiplepredictor model in addition to predictors that were statistically significantly different between the 2 study groups. In addition, the interaction effect between aprotinin and risk stratification based on the median Aristotle score was explored. Separate models for each level were considered if this effect was statistically significant. Power considerations allowed for multiple-predictor models for biochemical acute kidney failure and operative mortality outcomes. Odds ratios and corresponding $95 \%$ confidence intervals are presented for statistically significant categorical predictors. The incidence of neurologic outcomes was low, and hence the $\chi^{2}$ test was used to test the association between aprotinin and outcome if adverse outcomes were observed in at least 10 operations. The low incidence did not allow for repeated-measures analyses of multiple operations.

Statistical analyses were conducted with SAS statistical software (version 9.1; SAS, Cary, NC) and S-Plus (version 6.2; Insightful Corp, Seattle, Wash). All conclusions were made at the .05 level of significance. 
TABLE 2. Patient population: Demographics and operative predictors

\begin{tabular}{lccc}
\hline & $\begin{array}{c}\text { No } \\
\text { aprotinin }\end{array}$ & Aprotinin & $\boldsymbol{P}$ value \\
\hline Demographics & & & \\
No. of patients & 1083 & 1007 & \\
No. of operations & 1230 & 1251 & - \\
Age (y) & $3.64 \pm 4.75$ & $3.49 \pm 1.84$ & $.019^{*}$ \\
BSA & $0.57 \pm 0.41$ & $0.54 \pm 0.45$ & .084 \\
Male sex & $52.5 \%$ & $55.4 \%$ & .4 \\
Operative predictors & & & \\
Aristotle score & $7.23 \pm 2.57$ & $7.81 \pm 2.31$ & $<.001^{*}$ \\
Emergency status & 49 & 87 & $.011^{*}$ \\
Prior open CT & 338 & 362 & .42 \\
$\quad$ operations & & & \\
Preoperative & 202 & 211 & .77 \\
$\quad$ ventilator support & & & \\
Preoperative serum & $0.49 \pm 0.26$ & $0.52 \pm 0.39$ & .07 \\
$\quad$ creatinine level & & & \\
(mg/dL) & & & \\
DHCA & 107 & 86 & .09 \\
CPB time (min) & $124 \pm 67$ & $120 \pm 65$ & .067 \\
Aortic crossclamp & $58.9 \pm 45.2$ & $59.3 \pm 45.7$ & .82 \\
time (min) & & & \\
(n) & & &
\end{tabular}

$B S A$, Body surface area; $C T$, cardiothoracic; DHCA, deep hypothermic circulatory arrest; $C P B$, cardiopulmonary bypass. ${ }^{*}$ Significant.

\section{Results}

The demographic and operative predictors of the 2 patient cohorts are shown in Table 2. The patients who received aprotinin were younger than the patients who did not receive aprotinin. The aprotinin group contained more patients having an operation with emergency status. The Aristotle score of the patients who received aprotinin was higher than that of the patients who did not receive aprotinin. There was no significant difference between the groups in body surface area, CPB time, crossclamp time, use of circulatory arrest, preoperative serum creatinine level, prior open cardiothoracic operations, and preoperative ventilator support.

\section{Operative Mortality}

The mortality for the no-aprotinin group was $4.5 \%$, and in the aprotinin group it was $3.8 \%(P=.37$, Table 3$)$. Controlling for other patient risk factors in the multivariate analysis, there continued to be no association between aprotinin and operative mortality $(P=.508$, Table 4$)$. Significant predictors of operative mortality in this model were use of DHCA, preoperative ventilator support, longer CPB time, and smaller body surface area.

\section{Biochemical Acute Kidney Failure}

In the no-aprotinin group biochemical acute kidney failure occurred in $68(6.0 \%)$ of 1133 patients. In the aprotinin
TABLE 3. Results: Univariate analysis

\begin{tabular}{lccc}
\hline & $\begin{array}{c}\text { No } \\
\text { aprotinin }\end{array}$ & Aprotinin & $\boldsymbol{P}$ value \\
\hline Operative mortality & 55 & 47 & .37 \\
Biochemical acute kidney failure & 68 & 69 & .76 \\
Mean highest postoperative & $0.58 \pm 0.31$ & $0.6 \pm 0.33$ & .08 \\
$\quad$ serum creatinine (mg/dL) & & & \\
Temporary dialysis & 6 & 12 & .166 \\
Mortality caused by dialysis & 5 & 6 & $>.99$ \\
\hline
\end{tabular}

group this occurred in $69(5.7 \%)$ of 1210 patients $(P=.76)$. Data were incomplete regarding serum creatinine levels for $138(6.6 \%)$ patients, 97 in the no-aprotinin group and 41 in the aprotinin group. Controlling for other patient risk factors in the multivariate analysis, there was no association between aprotinin and biochemical acute kidney failure $(P=$ .77 , Table 5). Significant predictors of biochemical acute kidney failure in this model were use of DHCA, preoperative ventilator support, younger age, longer CPB time, and lower preoperative creatinine level.

A subanalysis was done in which patients were stratified into low- or high-risk groups by using the median Aristotle score (7.8) as the cutoff value. The interaction effect between aprotinin and risk category was not statistically significant $(P=.5)$, indicating that aprotinin did not have a differential effect on the outcome of biochemical acute kidney failure, depending on risk status.

\section{Temporary Dialysis}

In the no-aprotinin group $6(0.49 \%)$ of 1229 patients required temporary dialysis in the postoperative period. In the aprotinin group this was $12(0.96 \%)$ of $1251(P=.166)$. Temporary dialysis was associated with a $61 \%$ mortality

TABLE 4. Operative mortality: Multivariate analysis

\begin{tabular}{|c|c|c|c|}
\hline Predictor & $P$ value & Odds ratio & $\begin{array}{c}95 \% \text { Confidence } \\
\text { interval }\end{array}$ \\
\hline Aprotinin & .51 & 一 & 一 \\
\hline Emergency status & .079 & 一 & 一 \\
\hline DHCA & $<.001^{*}$ & 3.32 & $1.8-6.0$ \\
\hline $\begin{array}{l}\text { Preoperative } \\
\text { ventilator support }\end{array}$ & $<.001^{*}$ & 3.61 & $1.9-6.9$ \\
\hline Predictor & $P$ value & $\begin{array}{c}\text { No } \\
\text { operative } \\
\text { mortality }\end{array}$ & $\begin{array}{l}\text { Operative } \\
\text { mortality }\end{array}$ \\
\hline Age (y) & .29 & $3.5 \pm 4.8$ & $0.8 \pm 2.5$ \\
\hline BSA & $.008^{*}$ & $0.57 \pm 0.43$ & $0.28 \pm 0.22$ \\
\hline Aristotle score & .68 & $7.4 \pm 2.4$ & $9.7 \pm 3.3$ \\
\hline CPB time (min) & $<.001^{*}$ & $120 \pm 57$ & $175 \pm 102$ \\
\hline
\end{tabular}

$D H C A$, Deep hypothermic circulatory arrest; $B S A$, body surface area; $C P B$, cardiopulmonary bypass. *Significant. 
TABLE 5. Biochemical acute kidney failure: Multivariate analysis

\begin{tabular}{lccc}
\hline Predictor & P value & Odds ratio & $\begin{array}{c}\text { 95\% Confidence } \\
\text { interval }\end{array}$ \\
\hline Aprotinin & .77 & - & - \\
DHCA & $<.001^{*}$ & 3.05 & $1.68-5.53$ \\
Preoperative & $.003^{*}$ & 2.27 & $1.33-3.88$ \\
ventilator support & & & \\
\hline & & & Acute \\
& & No acute kidney & kidney \\
Predictor & $\boldsymbol{P}$ value & failure & failure \\
\hline Age (y) & $.017^{*}$ & $3.5 \pm 4.8$ & $2.0 \pm 3.9$ \\
Aristotle score & .063 & $7.5 \pm 2.4$ & $8.5 \pm 2.7$ \\
CPB time (min) & $<.001^{*}$ & $119 \pm 58$ & $153 \pm 73$ \\
Preoperative serum & $<.001^{*}$ & $0.51 \pm 0.3$ & $0.39 \pm 0.2$ \\
$\quad$ creatinine level & & & \\
$\quad$ (mg/dL) & & & \\
\hline
\end{tabular}

$\overline{D H C A}$, Deep hypothermic circulatory arrest; $C P B$, cardiopulmonary bypass. *Significant.

rate because 11 of these 18 patients died. It should be noted that all of these were very complex procedures; no patient who had a straightforward cardiac procedure had postoperative acute kidney failure. The mean Aristotle score of the no-aprotinin group to require dialysis was 7.7 , and the mean score in the aprotinin group requiring dialysis was 10.1. Mortality related to temporary dialysis was similar in both groups. There were $5(0.41 \%)$ deaths in dialyzed patients in the no-aprotinin group and $6(0.48 \%)$ deaths in dialyzed patients in the aprotinin group $(P>.99)$. There was no power for multivariable models beyond the 2 predictor models. Odds ratios for predictors are from the univariate model. Predictors of temporary dialysis were emergency status, use of DHCA, preoperative ventilator support, higher Aristotle score, longer CPB time, and higher preoperative serum creatinine level (Table 6). No patient required permanent dialysis.

\section{Neurologic Outcomes}

Our cardiac surgery database includes a category for neurologic complications, one of which is "postoperative newonset neurologic deficit persisting at discharge." In the no-aprotinin group this occurred in $3(0.24 \%)$ of 1230 patients, and in the aprotinin group this occurred in 3 $(0.24 \%)$ of 1251 patients. In the no-aprotinin group postoperative new-onset seizures occurred in $11(0.89 \%)$ of 1230 patients. In the aprotinin group these occurred in $14(1.1 \%)$ of 1251 patients $(P=.58)$. There was no statistically significant difference observed between the 2 groups for either neurologic outcome.

\section{Adverse Reactions and Re-exposures}

Two patients had a possible anaphylactic reaction to aprotinin. In both cases it was their first exposure to the drug,
TABLE 6. Postoperative temporary dialysis: Univariate analysis

\begin{tabular}{|c|c|c|c|}
\hline Predictor & $P$ value & Odds ratio & $\begin{array}{l}95 \% \text { Confidence } \\
\text { interval }\end{array}$ \\
\hline Aprotinin & .166 & - & - \\
\hline Emergency status & $<.001^{*}$ & 6.3 & $2.2-17.9$ \\
\hline DHCA & $<.001^{*}$ & 6.4 & $2.3-17.6$ \\
\hline $\begin{array}{l}\text { Preoperative } \\
\text { ventilator support }\end{array}$ & $<.001^{*}$ & 5.1 & $2.0-13.0$ \\
\hline Predictor & $P$ value & $\begin{array}{c}\text { No } \\
\text { temporary } \\
\text { dialysis } \\
\end{array}$ & $\begin{array}{c}\text { Temporary } \\
\text { dialysis } \\
\end{array}$ \\
\hline Age (y) & .09 & 一 & 一 \\
\hline Aristotle score & $<.001^{*}$ & $7.5 \pm 2.5$ & $9.3 \pm 2.6$ \\
\hline CPB time (min) & $<.001^{*}$ & $121 \pm 60$ & $196 \pm 114$ \\
\hline $\begin{array}{l}\text { Preoperative serum } \\
\text { creatinine level } \\
\text { (mg/dL) }\end{array}$ & $.031 *$ & $0.5 \pm 0.3$ & $0.9 \pm 0.7$ \\
\hline
\end{tabular}

$D H C A$, Deep hypothermic circulatory arrest; $C P B$, cardiopulmonary bypass. *Significant.

and neither patient had a reaction to the initial test dose. The first patient became hypotensive on aprotinin administration, with a decrease in systolic blood pressure from 110 to $50 \mathrm{~mm} \mathrm{Hg}$. Systolic blood pressure recovered to baseline after discontinuation of aprotinin and with administration of dopamine and fluids. The second patient had decreased lung compliance after aprotinin administration, necessitating discontinuation of aprotinin. The patient recovered to baseline pulmonary function after administration of epinephrine boluses and dopamine.

There were 91 patients who were re-exposed to aprotinin once within 1 year of their first exposure. Three additional patients were re-exposed more than once within a year of their exposure to aprotinin. No adverse events were associated with re-exposure to aprotinin in this series.

\section{Late Mortality}

As determined by means of Cox regression analysis, aprotinin had no influence on late mortality ( 24 deaths in the no-aprotinin group vs 10 deaths in the aprotinin group, $P=$ .078). This analysis was of course complicated by the separate time cohorts, with significantly more follow-up in the no-aprotinin group. However, this was counterbalanced by the relatively short mean time to late mortality in all groups. The mean time to late death in all groups was $1.7 \pm 2.3$ years (median, 0.77 years). In the no-aprotinin group mean time to late death was $2.23 \pm 2.49$ years (median, 1.3 years), and in the aprotinin group mean time to late death was $0.44 \pm 6.5$ years (median, 0.22 years). The mean follow-up time in the no-aprotinin group was $8.54 \pm 3.79$ years (median, 9.4 years), and in the aprotinin group the 
TABLE 7. Late mortality: Cox regression analysis (time to event outcome)

\begin{tabular}{|c|c|c|c|}
\hline Predictor & $P$ value & $\begin{array}{l}\text { Odds } \\
\text { ratio }\end{array}$ & $\begin{array}{c}\text { 95\% Confidence } \\
\text { interval }\end{array}$ \\
\hline Aprotinin & .78 & - & - \\
\hline $\begin{array}{l}\text { Preoperative } \\
\text { ventilatory support }\end{array}$ & $<.001^{*}$ & 4 & $2-8.1$ \\
\hline Emergency status & $<.001^{*}$ & 6.65 & $2.6-16.9$ \\
\hline $\begin{array}{c}\text { Prior open CT } \\
\text { operations }\end{array}$ & $.043^{*}$ & 2 & $1-4$ \\
\hline Predictor & $P$ value & $\begin{array}{c}\text { No late } \\
\text { mortality }\end{array}$ & $\begin{array}{c}\text { Late } \\
\text { mortality }\end{array}$ \\
\hline Aristotle Score & $<.001^{*}$ & $7.4 \pm 2.4$ & $8.6 \pm 2.0$ \\
\hline CPB time (min) & $<.001^{*}$ & $119 \pm 57$ & $149 \pm 62$ \\
\hline
\end{tabular}

$C T$, Cardiothoracic; $C P B$, cardiopulmonary bypass. *Significant.

mean follow-up was $3.47 \pm 1.99$ years (median, 3.3 years). Mean and median follow-up times in the aprotinin group encompass the mean and median times to late mortality. Table 7 shows the predictors of late mortality. These included preoperative ventilator support, preoperative emergency status, prior open cardiothoracic operation, higher Aristotle score, and longer CPB time.

\section{Limitations}

The primary limitation of this study was that the patients were not randomly assigned and were not contemporaneous. There might have been changes in the operative and postoperative protocols that affected the outcomes irrespective of aprotinin administration, thus creating an unintentional time-based selection bias in our study. However, a very important factor is that there was no selection bias for the use or nonuse of aprotinin. There was a distinct policy change in early 2000, and all patients undergoing CPB received aprotinin after that time. The bias between the 2 cohorts, which would actually factor against aprotinin for poorer outcomes in this group, are that the patients in the aprotinin group were slightly younger (3.49 vs 3.64 years) and had a significantly higher Aristotle score (7.81 vs 7.23). The Aristotle score has been validated as an indicator of postoperative mortality. ${ }^{19}$ In addition, there were more emergency cases in the aprotinin group (87 vs 49 ).

The analyses of aprotinin risk in relation to postoperative neurologic deficit and seizure are based on complication incidence, as recorded in our comprehensive cardiac surgery database. We found no statistically significant difference between the 2 groups for either outcome. However, these patients did not have routine postoperative examinations by a neurologist or routine postoperative neurologic imaging, and therefore this analysis is not comprehensive. Manifestations of neurologic injury can be subtle and might only become evident over time.

\section{Discussion}

This retrospective study of 2090 patients operated on between 1994 and 2006 was performed to assess the safety of aprotinin in pediatric patients undergoing CPB. This study was facilitated by a policy change to use aprotinin in all patients undergoing CPB in early 2000. The two 6-year cohorts of patients were compared.

The impetus for this study was the recent article, "The risk associated with aprotinin in cardiac surgery," by Mangano and associates. ${ }^{10}$ This was a propensity-adjusted observational study that reported that aprotinin use $(n=1295)$ was associated with a doubling of the risk of perioperative acute kidney failure and cerebral vascular accidents in patients undergoing CPB grafting. This study has elicited numerous letters to the editor and editorials and has called into question the use of aprotinin for patients undergoing CPB. ${ }^{12,14}$ Another report from Mangano and associates ${ }^{11}$ correlated the use of aprotinin $(n=1072)$ with a significantly increased late mortality in patients undergoing coronary artery bypass graft surgery. This report was also the subject of an editorial response. ${ }^{14}$ The primary questions raised regarding the Mangano studies are related to the reason the patients receive the drug therapy (aprotinin) in the first place and the influence of that selection bias on the patient data collection process and analysis and patient outcomes. ${ }^{12-14}$ Our review would indicate that for pediatric patients, the use of aprotinin $(\mathrm{n}=1251)$ was not associated with an increase in the risk of biochemical acute kidney failure, need for dialysis, or neurologic complications and also was not associated with increased risk of operative or late mortality. This was despite the fact that the aprotinin group of patients was statistically younger and had a higher Aristotle score.

One counterintuitive finding was that the preoperative serum creatinine level in the patients who had biochemical acute kidney failure was less preoperatively than the level in those patients who did not have biochemical acute kidney failure. The answer to why the lower serum creatinine value was a predictor of postoperative acute kidney failure is probably explained by the fact that younger children tended to have kidney failure ( 2.0 vs 3.5 years). The younger cohort of children has a lower muscle mass and therefore a lower serum creatinine level as normal baseline. Our definition of biochemical acute kidney failure as an increase in serum creatinine level to twice or more than the preoperative level uses each patient's preoperative serum creatinine level as his or her own control, a practice that is consistent with our (Children's Memorial Hospital) internal standard for the definition of acute kidney failure, as well as that of our cardiac surgery database (personal e-mail communication with J. P. Jacobs, Multi-Societal Pediatric and Congenital Cardiac Database Taskforce, 2007). In addition, it should be noted that although the preoperative serum creatinine values were lower in those children who had acute kidney failure, 
the respective values of 0.39 versus $0.51 \mathrm{mg} / \mathrm{dL}$, although statistically different, are not clinically different.

It should be clearly noted that our study was not performed to demonstrate the efficacy of aprotinin in pediatric patients. There have been numerous previous studies to indicate that aprotinin is indeed efficacious in pediatric patients undergoing CPB. A recent meta-analysis by Arnold and colleagues ${ }^{20}$ reported that aprotinin reduced the proportion of children who received red blood cell or whole blood transfusions during cardiac surgery by 33\%. Our own study published in 2003 demonstrated that with the use of aprotinin, children were exposed to 3 instead of 5 red blood cell units. Operative closure time was less (ie, 93 vs 127 minutes, a savings of 34 minutes). The Ann Arbor group in 1996 reported in a prospective, randomized, placebo-controlled, double-blind trial that aprotinin resulted in fewer exposures to bank-blood components and was also associated with a savings in the patient charges for blood components, operating room time, and duration of hospitalization. ${ }^{8}$ The group from Eggleston Children's Hospital in 1998 reported similar findings. ${ }^{9}$

A recent study at the University of California, San Francisco, evaluated the use or nonuse of aprotinin in patients undergoing the Norwood, Glenn, and Fontan procedures. The authors concluded the following: "The key point of these data is that we did not see evidence of clinical concern in this population of children with ... aprotinin. If anything our data support the safety of these drugs for use in children undergoing the repair of congenital cardiac defects." ${ }^{21}$ The Milwaukee group, in particular, has demonstrated the utility and safety of aprotinin use and reuse in pediatric patients undergoing cardiothoracic procedures. ${ }^{22,23}$ They concluded that the risk of hypersensitivity reactions to aprotinin is low (approximately 1\%), even with multiple exposures to the medication. Our analysis of the risk of re-exposure confirms the Milwaukee analysis; we had no adverse responses in 94 patients re-exposed within 1 year.

In our study of 2090 pediatric patients undergoing CPB, there was no association between the use of high-dose aprotinin and operative or late mortality, biochemical acute kidney failure, need for temporary dialysis, or neurologic complications. Given the previous studies demonstrating its efficacy, we continue to use aprotinin in all pediatric patients undergoing $\mathrm{CPB}$.

\section{References}

1. Approval of aprotinin [press release]. Washington (DC): US Food and Drug Administration; December 30, 1993. Available at: http://www. fda.gov/bbs/topics/NEWS/NEW00453.html.

2. Levy JH, Pifarre R, Schaff HV, et al. A multicenter, double-blind, placebo-controlled trial of aprotinin for reducing blood loss and the requirement for donor-blood transfusion in patients undergoing repeat coronary artery bypass grafting. Circulation. 1995;92:2236-44.

3. Cosgrove DM 3rd, Heric B, Lytle BW, et al. Aprotinin therapy for reoperative myocardial revascularization: a placebo-controlled study. Ann Thorac Surg. 1992;54:1031-6.
4. Sedrakyan A, Treasure T, Elefteriades JA. Effect of aprotinin on clinical outcomes in coronary artery bypass graft surgery: a systematic review and meta-analysis of randomized clinical trials. J Thorac Cardiovasc Surg. 2004;128:442-8.

5. Kozik DJ, Tweddell JS. Characterizing the inflammatory response to cardiopulmonary bypass in children. Ann Thorac Surg. 2006; 81(suppl):S2347-54.

6. Hill GE, Alonso A, Spurzem JR, Stammers AH, Robbins RA. Aprotinin and methylprednisolone equally blunt cardiopulmonary bypassinduced inflammation in humans. J Thorac Cardiovasc Surg. 1995; 110:1658-62.

7. Costello JM, Backer CL, de Hoyos A, Binns HJ, Mavroudis C. Aprotinin reduces operative closure time and blood product use after pediatric bypass. Ann Thorac Surg. 2003;75:1261-6.

8. D'Errico CC, Shayevitz JR, Martindale SJ, Mosca RS, Bove EL. The efficacy and cost of aprotinin in children undergoing reoperative open heart surgery. Anesth Analg. 1996;83:1193-9.

9. Miller BE, Tosone SR, Tam VK, et al. Hematologic and economic impact of aprotinin in reoperative pediatric cardiac operations. Ann Thorac Surg. 1998;66:535-40.

10. Mangano DT, Tudor IC, Dietzel C, Multicenter Study of Perioperative Ischemia Research Group, Ischemia Research and Education Foundation. The risk associated with aprotinin in cardiac surgery. $N$ Engl J Med. 2006;354:353-65.

11. Mangano DT, Miao Y, Vuylstekc A, et al. Mortality associated with aprotinin during 5 years following coronary artery bypass graft surgery. JAMA. 2007;297:471-9.

12. Levy JH, Ramsay JG, Guyton RA. Aprotinin in cardiac surgery [letter to the editor]. N Engl J Med. 2006;354:1953-7.

13. Royston D, van Haaften N, De Vooght P. Aprotinin; friend or foe? Eur $J$ Anesthesiol. 2007;24:6-14.

14. Ferguson TB Jr. Aprotinin-are there lessons learned? JAMA. 2007; 297:471-9.

15. Jacobs JP, Mavroudis C, Jacobs ML, Joint EACTS-STS Congenital Database Committee. What is operative mortality? Defining death in a surgical registry database: a report of the STS Congenital Database Taskforce and the Joint EACTS-STS Congenital Database Committee. Ann Thorac Surg. 2006;81:1937-41.

16. Kincaid EH, Ashburn DA, Hoyle JR, Reichert MG, Hammon JW, Kon ND. Does the combination of aprotinin and angiotensin-converting enzyme inhibitor cause renal failure after cardiac surgery? Ann Thorac Surg. 2005;80:1388-93.

17. Therneau TM, Grambsch PM. Modeling survival data: extending the Cox model. New York: Springer; 2000.

18. Liang KY, Zeger SL. Longitudinal data analysis using generalized linear models. Biometrika. 1986;73:13-22.

19. O'Brien SM, Clarke DP, Jacobs JP, et al. Accuracy of the Aristotle basic complexity score for classifying the mortality and morbidity potential of congenital heart surgery procedures. Paper presented at: 43rd Annual Meeting of the Society of Thoracic Surgeons; January 29, 2007; San Diego, Calif.

20. Arnold DM, Fergusson DA, Chan AK, et al. Avoiding transfusions in children undergoing cardiac surgery: a meta-analysis of randomized trials of Aprotinin. Anesth Analg. 2006;102:731-7.

21. Skidmore KL, Azakie A. Aprotinin and Sevoflurane do not affect renal function during single-ventricle palliative surgery [letter to the editor] Anesth Analg. 2006;103:1614-5.

22. Jaquiss RD, Chanayem NS, Zacharisen MC, et al. Safety of aprotinin use and re-use in pediatric cardiothoracic surgery. Circulation. 2002 106(suppl I):I90-4.

23. Tweddell JS, Berger S, Frommelt PC, et al. Aprotinin improves outcome of single-ventricle palliation. Ann Thorac Surg. 1996;62 1329-36.

\section{Discussion}

Dr James S. Tweddell (Milwaukee, Wis). That was an excellent presentation, Carl, as usual. This is a timely contribution from the group at Children's Memorial Hospital. The authors looked at their entire experience with aprotinin, a period of 6 years, and compared this with the previous 6-year period. Just over 2000 patients are 
included, pretty much evenly divided between the use and nonuse of aprotinin, making this the largest single-center report concerning aprotinin use in pediatrics by far.

Despite an increase in case complexity in the most recent aprotinin cohort, there is no difference in mortality or renal impairment, suggesting that aprotinin use is safe in this age group. Incidentally, our aprotinin use policy is identical to yours.

The limitations of this study have been acknowledged by the authors and most importantly include the comparison of noncontemporary patient groups. I would contend that this is a form of selection bias.

This study begins in 1994, and just for some perspective, in 1994, the sitcom "Friends" premiered on NBC, Netscape 1.0 was released, and George Bush was unequivocally elected governor of Texas. Times have changed.

Since 1994, we have seen some important changes in various aspects of preoperative, intraoperative, and postoperative management of patients with congential heart disease, including some pioneered from your institution.

Taking the devil's advocate position, one could argue that your most recent results, which are excellent, would have been even better if you had not used aprotinin. Therefore my comments and questions are really directed at potential ways around this timebias issue.

The most recent studies from the Ischemia Research and Education Foundation purported to show that aprotinin use was associated with a significantly increased risk of complications in adults-myocardial infarction, stroke and renal failure-in patients not having complex operations. Could you or did you analyze the effect of aprotinin risk within risk stratification categories? You could divide your patient population above and below the 50th percentile Aristotle score, for example, and this would be a way to match aprotinin use and risk stratification. Perhaps the risk/benefit ratio of aprotinin is favorable for high-risk patients but not so for low-risk patients. You had these data, and I think this would make an additional excellent analysis, and I would like to hear your comments on that.

Dr Backer. We did try to look at risk stratification based on Aristotle scores. We were particularly interested in the group of patients who required postoperative temporary dialysis because those were the patients who clearly had substantial kidney failure. The mean Aristotle score in the no-aprotinin group that required dialysis was 7.67. All of these patients had difficult operations, such as tricuspid valve replacement after heart transplantation and disrupted aortic annulus with emergency operation.

The mean Aristotle score in the aprotinin group that required dialysis was 10.08 , a high score that was almost statistically higher than that of the other group. If you look at this patient population, again, all these patients were at very high risk. There was a Fontan conversion with an Aristotle score of 12, there were 2 patients in heart transplantation status after Fontan conversion, there was a transplantation after a ventricular assist device, and there was a patient with a switch with an intramural coronary artery who was on extracorporeal membrane oxygenation. We did not have any patient who had, for example, a straightforward ventricular septal defect closure and then had kidney failure.

The other part of the data to look at is the multivariate analysis of biochemical acute kidney failure. The mean Aristotle score in the group without acute kidney failure was 7.5, and in those with acute kidney failure, it was 8.5. The Aristotle score only trended to significance, with a $P$ value of .063 . In the patients who required postoperative temporary dialysis, where the Aristotle score was statistically significant $(P<.001)$, it was 7.5 in the patients who did not require dialysis and 9.3 in the patients who did require postoperative dialysis.

However, your point is well taken, and we could incorporate risk stratification as a specific subanalysis of the data.

Dr Tweddell. Those are certainly compelling data, but I would suggest that it might be worthwhile performing the analysis.

Concerning the incidence of renal insufficiency, as determined based on chemical data, interestingly, the preoperative creatinine level was less in patients who sustained postoperative biochemical renal dysfunction, at least the way you defined it. This really causes me to question the validity of this definition. I know you used the somewhat arbitrary definition that was developed by the Society of Thoracic Surgeons in the multidisciplinary working group, and the purpose of that is really to allow some multiinstitutional comparisons. Because this is a single-institution study and because I am certain Children's Memorial has age-specific creatinine ranges of normalcy, you could actually analyze that separately. I think that would be important because that is obviously an important conclusion of your article.

Dr Backer. That is a good point. We discussed with our pediatric nephrologists what definition we should use for acute kidney failure. One of the problems with taking the absolute value is that if a sick patient's preoperative creatinine level is increased, for instance to 1.5 times normal value, then a postoperative level of 1.5 times normal value is not a change. The Society of Thoracic Surgeons-European Association for Cardiothoracic Surgery definition of acute kidney failure is a doubling of the creatinine level compared with the preoperative value or a level that is 1.5 times normal value, but the doubling seemed to take precedence for this reason. Our pediatric nephrologists believed that using doubling of the preoperative creatinine level as our definition would "widen the net," so that we would not miss patients with borderline postoperative kidney failure.

You noted, as we did in the article, that in the multivariate analysis there was the counterintuitive finding that the preoperative creatinine level was $0.51 \mathrm{mg} / \mathrm{dL}$ in the patients who did not have acute kidney failure, and it was $0.39 \mathrm{mg} / \mathrm{dL}$ in the patients who did have acute kidney failure. The question here is whether a creatinine level of 0.5 versus $0.4 \mathrm{mg} / \mathrm{dL}$ is actually clinically relevant.

In contrast, for those patients who required postoperative temporary dialysis, the association of preoperative creatinine level was significant, with a $P$ value of .03 . The patients who did not require temporary dialysis had a mean preoperative creatinine level of 0.5 $\mathrm{mg} / \mathrm{dL}$. If they did require temporary dialysis, the mean preoperative value was $0.9 \mathrm{mg} / \mathrm{dL}$. Therefore in the subgroup with kidney failure requiring postoperative dialysis, they did have a higher preoperative creatinine level, almost twice as high as before the operation.

Dr Tweddell. I guess I was suggesting that you use the most inclusive definition because that is important to rule it out. Also, I think that is more important than the application of dialysis because that is really measuring a clinical response and not necessarily biochemical evidence of renal failure. 
I have just a couple of other questions. Aprotinin is a strong inhibitor of bradykinin generation. Bradykinin is implicated in perioperative neurologic injury. Did you look at neurologic complications, seizures, and new deficits?

Dr Backer. We do have neurologic data in our database, although we did not specifically look at it. We could go back and analyze that relatively easily. However, we do not currently have late follow-up on neurologic outcomes.

Dr Tweddell. Have you had incidents of anaphylaxis, and how do you re-expose? Do you re-expose patients within a year?

Dr Backer. We relied on your article in Circulation that suggested that it was safe to redose aprotinin. The main group undergoing re-exposure within a year consisted of those who had the Norwood procedure with aprotinin and then within 4 to 6 months had the bidirectional Glenn procedure. Initially, our anesthesiologists were hesitant to use aprotinin during the Glenn procedure. Your article came out with the less than $1 \%$ anaphylaxis, and therefore currently, we administer aprotinin at the Norwood and Glenn procedures, irrespective of whether they have their Glenn procedure at 3.5, 4, or 6 months, and again at the Fontan procedure.

Our primary safety protocol is that every patient gets a test dose of aprotinin, and we wait to do that until the arterial line and the central line are in. We have had one patient who had significant hypotension related to the aprotinin-just one patient out of this entire group.

Dr Tweddell. Obviously there are no indications for aprotinin use in pediatrics, but there is now a black box warning about re-exposure within a year.

Dr Backer. Yes.
Dr Tweddell. It is one thing to use it when there is not an indication, and it is another thing to use it against a black box warning. I wonder if you have changed your policy at all? I think we are struggling with that as well.

Dr Backer. We have not changed our policy. The Friday before I came here, I did a reoperation to do a shunt revision on a patient after the Norwood procedure. We had used aprotinin 10 days earlier, and I used aprotinin again, and nothing happened. Granted, it is only 1 patient.

Dr Tweddell. My last and final question is whether you think we need a postmarket randomized controlled trial in pediatrics to approve efficacy and safety?

Dr Backer. Well, I think in an ideal world that would be a good thing. I think if there are enough questions about this and if centers are unable to use aprotinin because of the negative press and because of parental concerns, a randomized prospective study might be the only way we are going to prove its safety. There have been several studies that have showed efficacy, and we could look at efficacy and safety in the prospective trial. Specifically, we could look at kidney function and even do some more in-depth analysis, such as postoperative GFR and postoperative urine collections for creatinine clearance.

Dr Tweddell. Thank you. Excellent article.

Dr Kenneth G. Warner (Boston, Mass). Did you see any increased incidence of thrombotic complications in the aprotinin group, such as strokes, premature closure of fenestrations, and deep vein thrombosis?

Dr Backer. No. I mean, we have almost no thrombosis in our patients and that was not-we did not see that. 